\title{
PROBLEMS OF GAS DYNAMICS IN PLANETARY NEBULAE
}

\author{
F. D. KAHN \\ (Dept. of Astronomy, University of Manchester, England)
}

\begin{abstract}
The expansion of a typical planetary nebula is studied by means of a simple model, with spherical symmetry. It is shown that there is a build-up of radiation pressure, due to Lyman- $\alpha$ photons trapped in the nebula, and that this causes the initial acceleration outwards. After some $1.5 \times 10^{11} \mathrm{sec}$ the nebula will have a radius of the order of $10^{17} \mathrm{~cm}$, and an expansion velocity of about $2 \times 10^{6} \mathrm{~cm} / \mathrm{sec}$. At roughly this time the dynamical effects of radiation pressure begin to be superseded by those of the recoil pressure at the ionization front, which continues to dominate until the nebula is fully ionized.

Finally some reasons are considered why most nebulae do not have spherically symmetrical shapes, but why so often they appear to be ring-like or butterfly-shaped.
\end{abstract}

\section{Introduction}

The motion of the gas in a planetary nebula is influenced by the pressure of the hot, ionized gas, by the pressure of the Lyman- $\alpha$ radiation trapped in the nebula, and by the heating of the newly ionized gas at the front of the ionized region. In this paper no account will be taken of possible hydromagnetic effects, for there is little reason, from either observation or theory, to believe that magnetic fields of any consequence can exist in planetary nebulae

\section{Some Simple Properties of H II Regions}

The luminous part of a planetary nebula is an Hil region, in which the hydrogen is kept almost fully ionized by the radiation of the central star. The work of Seaton and his group (Seaton, 1966; Harman and Seaton, 1966; and other references cited there) has led to a good description of the properties of such stars, and Seaton's data will be used here. During the evolution of a nebula the luminosity $L_{*}$ of the typical central star varies between $2 \times 10^{36}$ and $4 \times 10^{37} \mathrm{erg} / \mathrm{sec}$, and its surface temperature $T_{*}$ between $4 \times 10^{4}$ and $10^{5}{ }^{\circ} \mathrm{K}$.

The most important parameter is $I_{*}$, the rate of production of photons, by the central star, in the Lyman continuum. If the star radiates like a black body in the far ultraviolet (see, e.g., Gebbie and Seaton, 1963), then $I_{*}$ is related to $L_{*}$ by

$$
I_{*}=i\left(T_{*}\right) L_{*}
$$

and $i\left(T_{*}\right)$ varies only slowly with the temperature $T_{*}$ of the star. For $T_{*}=4 \times 10^{4}{ }^{\circ} \mathrm{K}$, 
$i\left(T_{*}\right)=1 \cdot 2 \times 10^{10}$ photons $/ \mathrm{erg}$; between $T_{*}=6 \times 10^{4} \mathrm{~K}$ and $10^{5}{ }^{\circ} \mathrm{K}, i\left(T_{*}\right)$ is virtually constant, at $1.8 \times 10^{10}$ photons/erg. A typical value for $I_{*}$ is therefore $10^{47}$ photons/sec.

Now hydrogen is by far the most important constituent of the nebula. It may be assumed that, in the early phases of its evolution, the ionized portion of the nebula is entirely enclosed in a shell of non ionized hydrogen. Almost all the Ly-c photons are absorbed in the HiI region, and go to balance the recombinations of protons and electrons there. These statements will be checked later. For the present, let $n_{i}$ equal the proton and the electron number density in the ionized region, which we take to be a sphere of radius $R$, and in which the ionized gas has a temperature $T_{i}=10^{4}{ }^{\circ} \mathrm{K}$.

The recombination of a proton and an electron to the ground state produces another Ly-c photon. Thus Ly-c photons are lost only when recombinations occur to excited states, so that, in a first approximation,

$$
I_{*}=\frac{4 \pi}{3} \beta n_{i}^{2} R^{3} .
$$

At $10^{4}{ }^{\circ} \mathrm{K}$ the recombination coefficient to all excited states of the $\mathrm{H}$ atom is $\beta \approx 2 \times 10^{-13} \mathrm{~cm}^{3} / \mathrm{sec}$. Equation (2) leads to an estimate of the total mass of ionized gas

$$
M_{i}=\frac{4 \pi}{3} n_{i} m_{\mathrm{H}} R^{3}=\left(\begin{array}{cc}
4 \pi & I_{*} \\
3 & \beta
\end{array}\right)^{1 / 2} m_{\mathrm{H}} R^{3 / 2} \approx 2.6 \times 10^{5}\left(\begin{array}{c}
L_{*} \\
\beta
\end{array}\right)^{1 / 2} m_{\mathrm{H}} R^{3 / 2},
$$

where $m_{\mathrm{H}}$ is the mass of the $\mathrm{H}$ atom. If $L_{*}$ is held fixed, then $M_{i}$ varies like $R^{3 / 2}$. As $R$ increases, the mass of ionized gas increases, presumably at the expense of the nonionized gas in the shell which surrounds it. If $L_{*}$ and $R$ increase together, $M_{i}$ increases still faster.

Now a fraction $\varepsilon$ of the available Ly-c photons is spent in ionizing the atomic hydrogen drawn into the $\mathrm{H}$ II region as the radius $R$ increases. It will now be shown that $\varepsilon$ remains small throughout. From Equation (3), with $L_{*}$ fixed,

$$
\begin{aligned}
& \dot{M}_{i} \\
& M_{i}
\end{aligned}=\begin{aligned}
& 3 \dot{R} \\
& 2 R
\end{aligned} .
$$

The number of new protons entering per unit time is $\dot{M}_{i} / m_{\mathrm{H}}$, so that

$$
\varepsilon I_{*}=\dot{M}_{i} / m_{\mathrm{H}}=2 \pi n_{i} R^{2} \dot{R} .
$$

From Equations (2) and (5), then

$$
\varepsilon=\left(\beta n_{i}\right)^{-1}(\dot{R} / R) .
$$

$\left(\beta n_{i}\right)^{-1}$ is the characteristic time for the recombination of a proton and an electron, about $10^{9} \mathrm{sec}$ with a reasonable value of $n_{i} . R / \dot{R}$ is the characteristic expansion time of the nebula, of the order of $10^{11} \mathrm{sec}$. Thus $\varepsilon$ is of the order of 0.01 . Alternatively, it 
may also be deduced from Equations (2) and (5) that

$$
\varepsilon=\left(\frac{4 \pi}{3 \beta I_{*}}\right)^{1 / 2} \dot{R} R^{1 / 2} \approx 6 \times 10^{-18} \dot{R} R^{1 / 2},
$$

with our typical value for $I_{*}$. Once again it is clear that, even though $\varepsilon$ increases as the nebula expands, it always remains a small quantity. These conclusions will not be qualitatively changed if $I_{*}$ varies with time.

A good approximation, usually made, is that a Ly- $\alpha$ photon will eventually be emitted in the Hil region for every Ly-c photon absorbed there (see, e.g., Aller, 1956). The rate of production of radiant energy in the Ly- $\alpha$ line thus becomes

$$
L_{\alpha}=I_{*} \chi_{\alpha}=i\left(T_{*}\right) \chi_{\alpha} L_{*},
$$

where $\chi_{\alpha}=10 \cdot 15 \mathrm{eV}$ is the energy of the Ly- $\alpha$ photon. With the quoted value for $i\left(T_{*}\right)$

$$
L_{\alpha} \approx 0 \cdot 3 L_{*}
$$

as long as the temperature $T_{*}$ lies between $5 \times 10^{4}{ }^{\circ} \mathrm{K}$ and $10^{5}{ }^{\circ} \mathrm{K}$.

\section{The Transfer of Lyman- $\alpha$ Radiation}

The Ly- $\alpha$ radiation cannot escape freely from the HII region, for there will always be a small proportion of atomic hydrogen present. In fact the ionization front is usually found at a distance from the central star such that

$$
\tau_{l}=\int_{0}^{R} \kappa_{l} \rho_{i}(1-x) \mathrm{d} r \approx 10 \text { or } 20,
$$

where $\kappa_{l} \approx 3.6 \times 10^{6} \mathrm{~cm}^{2} / \mathrm{gm}$ is the opacity of atomic hydrogen at the Lyman limit, $\rho_{i}$ is the density by mass of $\left(\mathrm{H}^{+}+\mathrm{H}\right)$, and $x$ is the fractional ionization within the $\mathrm{HII}$ region (see, e.g., Mathews, 1965). There should therefore be between $3 \times 10^{-6}$ and $5 \times 10^{-6} \mathrm{gm} / \mathrm{cm}^{2}$ of atomic hydrogen along a line between the central star and the ionization front.

In the absence of thermal motion, atomic hydrogen has an opacity $\varpi /\left(u^{2}+\eta^{2}\right)$ at frequency $v=v_{\alpha}-u$, where $\varpi=10^{29} \mathrm{~cm}^{2} \mathrm{gm}^{-1} \mathrm{~s}^{-2}, \eta=2.5 \times 10^{7} \mathrm{~s}^{-1}$, and $v_{\alpha}=$ $2.5 \times 10^{15} \mathrm{~s}^{-1}$, the frequency of Ly- $\alpha$. In the wings of Ly- $\alpha$ the Hil region will therefore be opaque out to a frequency shift given by

$$
\begin{aligned}
u^{2} & \approx 5 \times 10^{-6} \varpi \approx 5 \times 10^{23} \mathrm{~s}^{-2}, \text { or } \\
u & \approx \pm 7 \times 10^{11} \mathrm{~s}^{-1} .
\end{aligned}
$$

The non-ionized jacket around the HII region is even more opaque. It has a surface density

$$
\sigma=M / 4 \pi R^{2}
$$


and if $M=10^{33} \mathrm{gm}$ and $R=10^{17} \mathrm{~cm}$, then $\sigma \approx 10^{-2} \mathrm{gm} / \mathrm{cm}^{2}$, larger by some 3 orders of magnitude than the mass of atomic hydrogen along a unit column from the central star to the I-front. A photon with frequency close to $v_{\alpha}$ therefore cannot escape from the nebula, but the indefinite build-up of radiation density is prevented by the gradual drift of the photons in frequency. There are two important mechanisms:

(i) The photons are essentially trapped in an expanding enclosure. By a classical physical result their frequency then varies inversely as the linear scale of the enclosure, at a rate given by

$$
\dot{u}=v_{\alpha} \dot{R} / R
$$

The rate of change of $J$ due to this red-shift (rs) mechanism is

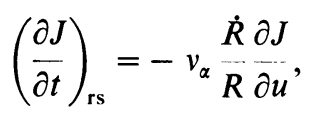

where $J(u) \mathrm{d} u$ is the photon density between frequencies $v_{\alpha}-u$ and $v_{\alpha}-u-\mathrm{d} u$.

(ii) The photons are scattered by the $\mathrm{H}$ atoms in the $\mathrm{HII}$ region. The scattering coefficient depends on frequency and is determined by Doppler and by natural line broadening, according to the formula

$$
\begin{aligned}
& p\left(u, u^{\prime}\right)=\frac{3 \varpi \rho_{\mathrm{H}} c}{8 \sqrt{2 \pi} U} \int_{0}^{\pi} \int_{-\infty}^{\infty} \frac{1+\cos ^{2} \theta}{u^{\prime \prime 2}+\eta^{2}} \exp \left[-\frac{1}{2 U^{2} \sin ^{2} \theta}\right. \\
& \left.\quad \times\left\{\left(u-u^{\prime \prime}\right)^{2}-2\left(u-u^{\prime \prime}\right)\left(u^{\prime}-u^{\prime \prime}\right) \cos \theta+\left(u^{\prime}-u^{\prime \prime}\right)^{2}\right\}\right] \mathrm{d} u^{\prime \prime} \mathrm{d} \theta,
\end{aligned}
$$

which is easily proved. $p\left(u, u^{\prime}\right) \mathrm{d} u^{\prime}$ is the probability per unit time, that a photon with frequency shift $u$ should be scattered into the range of frequency shift $u^{\prime}, u^{\prime}+\mathrm{d} u^{\prime}$;

$$
U=\frac{v_{\alpha}}{c} \sqrt{\frac{k T_{i}}{m_{\mathrm{H}}}} \approx 8 \times 10^{10} \mathrm{~s}^{-1}, \quad \text { when } T_{i}=10^{4}{ }^{\circ} \mathrm{K}
$$

and $\rho_{\mathrm{H}}$ is the density, by mass, of atomic hydrogen.

The redistribution of photons in frequency occurs at a rate

$$
\begin{aligned}
\frac{\partial J(u)}{\partial t} & =\int_{-\infty}^{\infty}\left\{p\left(u^{\prime}, u\right) J\left(u^{\prime}\right)-p\left(u, u^{\prime}\right) J(u)\right\} \mathrm{d} u^{\prime} \\
& =\int_{-\infty}^{\infty} p\left(u^{\prime}, u\right)\left\{J\left(u^{\prime}\right)-J(u)\right\} \mathrm{d} u^{\prime}
\end{aligned}
$$

and the second step in these relations follows because $p$ is symmetrical in $u$ and $u^{\prime}$. 
When the spectrum is flat, $J\left(u^{\prime}\right)=J(u)$ for all pairs of $u$ and $u^{\prime}$, and no net redistribution occurs.

This observation eases the calculation that follows. During the early phases of the evolution of a nebula, the Ly- $\alpha$ line is spread far out into the wings, and the spectrum close to $v_{\alpha}$ will be rather flat. Thus no additional change is introduced into the calculated profile even when the transfer near $v_{\alpha}$ is estimated by the correct formula, rather than by the approximate formula that really holds only in the wings of the line. The scattering in the wings is coherent, except for small Doppler shifts due to the thermal motion of the scattering atoms. It is easier to find suitable approximate formulae directly, rather than to derive them from (10).

Let a photon come from $P$, and let it be scattered at $O$ into the direction $O P^{\prime}$, such that there is an angle $\theta$ between $P O$ produced and $O P^{\prime}$. Let $O Q$ be the bisector of the angle $P O P^{\prime}$. Let the atom that scatters the photon have a velocity component $v$ parallel to $O Q$. Then it may be shown that an incoming photon, having frequency $v$, relative to the laboratory frame of reference, is scattered with frequency $v\{1+(2 v / c) \sin (\theta / 2)\}$ relative to that frame. The frequency change is

$$
\delta u=-2 v_{c}^{v} \cdot \sin { }_{2}^{\theta} \approx-2 v_{\alpha}{ }_{c}^{v} \sin { }_{2}^{\theta} \text {. }
$$

Taking averages over all values of $v$

$$
\langle\delta u\rangle_{v}=0
$$

and

$$
\left\langle\delta u^{2}\right\rangle_{v}=\frac{4 v_{\alpha}^{2}}{c^{2}} \frac{k T_{i}}{m_{\mathrm{H}}} \sin ^{2}{ }_{2} \text {. }
$$

Scattering angles are distributed symmetrically with respect to $\theta=\pi / 2$, so that

$$
\left\langle\left\langle\delta u^{2}\right\rangle_{v}\right\rangle_{\theta}=\frac{2 v_{\alpha}^{2}}{c^{2}}\left(\frac{k T_{i}}{m_{\mathrm{H}}}\right) \equiv 2 U^{2},
$$

and $U \approx 1 \cdot 1 \times 10^{11} \mathrm{~s}^{-1}$, with $T_{i}=10^{4}{ }^{\circ} \mathrm{K}$.

Apparently then, in the wings of Ly- $\alpha$,

$$
p\left(u, u^{\prime}\right)=\frac{\varpi \rho_{\mathrm{H}} c}{u^{2}} \phi\left(u^{\prime}-u\right)
$$

where $\phi$ is a symmetrical probability distribution, normalized to unity, with variance $U^{2}$. Since $\eta^{2} \ll U^{2}$ it is safe to neglect $\eta^{2}$ in the denominator. But this form for $p\left(u, u^{\prime}\right)$ is not symmetrical in $u$ and $u^{\prime}$, as it ought to be. The correct approximation is therefore

$$
p\left(u, u^{\prime}\right)=\frac{\varpi \rho_{\mathrm{H}} c}{2}\left(\frac{1}{u^{2}}+\frac{1}{u^{\prime 2}}\right) \phi\left(u^{\prime}-u\right),
$$


and Equation (11) becomes

$$
\frac{\partial J(u)}{\partial t}=\frac{\varpi \rho_{\mathrm{H}} c}{2} \int_{-\infty}^{\infty}\left(\frac{1}{u^{2}}+\frac{1}{u^{\prime 2}}\right) \phi\left(u^{\prime}-u\right)\left[J\left(u^{\prime}\right)-J(u)\right] d u^{\prime} .
$$

In the wings individual steps in frequency are small compared with the frequency difference from the line centre, so that one may expand under the integral sign, and find

$$
\begin{aligned}
& \frac{\partial J(u)}{\partial t}=\frac{\varpi \rho_{\mathrm{H}} c}{2} \int_{-\infty}^{\infty}\left[\frac{2}{u^{2}}-\frac{2\left(u^{\prime}-u\right)}{u^{3}}\right] \phi\left(u^{\prime}-u\right) \times \\
& {\left[\left(u^{\prime}-u\right) \frac{\partial J}{\partial u}+\frac{1}{2}\left(u^{\prime}-u\right)^{2} \begin{array}{l}
\partial^{2} J \\
\partial u^{2}
\end{array}\right] \mathrm{d} u^{\prime}}
\end{aligned}
$$

to second order. No contributions arise from terms which are linear in $\left(u^{\prime}-u\right)$, and to second order

$$
\begin{aligned}
\left(\begin{array}{c}
\partial J(u) \\
\partial t
\end{array}\right)_{\mathrm{sc}} & =\frac{\varpi \rho_{\mathrm{H}} c}{2}\left[-\frac{2 \partial J}{u^{3} \partial u}+\frac{1 \partial^{2} J}{u^{2} \partial u^{2}}\right] \int_{-\infty}^{\infty}\left(u^{\prime}-u\right)^{2} \phi\left(u^{\prime}-u\right) \mathrm{d} u^{\prime} \\
& =\varpi \rho_{\mathrm{H}} c U^{2} \frac{\partial}{\partial u}\left(\begin{array}{c}
1 \partial J \\
u^{2} \partial u
\end{array}\right) .
\end{aligned}
$$

This is the rate of change of photon density due to the scattering mechanism.

(iii) The photons leak through the atomic hydrogen in which they are trapped. The $\mathrm{H}$ II interior region of the nebula is much less opaque than the non-ionized shell. The interior therefore fills up to a uniform photon density $J(u)$ at any frequency. A gradient of photon density $J(u) / l$ will be set up across the thickness $l$ of the shell. If $\rho_{\mathbf{H}}$ is the density of atomic hydrogen there, its optical depth at frequency $v_{\alpha}-u$ is $\varpi \rho_{\mathrm{H}} l / u^{2}$ and the photon flux per unit frequency interval is

$$
F(u)=\frac{c J u^{2}}{3 \varpi \rho_{\mathrm{H}} l} \equiv \frac{c J u^{2}}{3 \varpi \sigma} .
$$

A sphere of radius $R$ contains $(4 \pi / 3) R^{3} J(u)$ photons per unit frequency interval. The rate of leakage of photons is therefore

and so

$$
{ }_{3}^{4 \pi} R^{3}\left(\frac{\partial J}{\partial t}\right)_{l}=-4 \pi R^{2} F(u)
$$

$$
\left(\frac{\partial J}{\partial t}\right)_{l}=-\frac{c u^{2}}{\varpi \sigma R} J(u)
$$


Now combine Equations (9), (19) and (21), and in Equation (19) set $\rho_{\mathrm{H}}=\rho_{i}(1-x)$, the density of atomic hydrogen in the HII region. In a steady state

$$
-v_{\alpha} \frac{\dot{R} \mathrm{~d} J}{R \mathrm{~d} u}+\varpi \rho_{i}(1-x) c U^{2} \frac{\mathrm{d}}{\mathrm{d} u}\left(\frac{1 \mathrm{~d} J}{u^{2} \mathrm{~d} u}\right)-\frac{c u^{2} J}{\varpi \sigma R}+L_{\alpha} \delta(u)=0
$$

The last term describes the rate at which Ly- $\alpha$ radiation is generated in the $\mathrm{H}_{\text {II }}$ region. Equation (22) is solved by setting $u^{2} \mathrm{~d} u \equiv \mathrm{d} z$, and then its solutions have the form $A_{1} e^{\lambda_{1} z}+A_{2} e^{-\lambda_{2} z}$, where $\lambda_{1}$ and $\lambda_{2}$ are both positive. Only a finite amount of energy can be trapped, so that both the solutions for positive and for negative $z$ must tend to zero as $|z|$, and therefore as $|u|$, tend to infinity. The solutions are respectively proportional to $e^{\lambda_{1} z}$ and $\mathrm{e}^{-\lambda_{2} z}$ for $u<0$ (the blue side) and for $u>0$ (the red side). Values of $\lambda_{1}$ and $\lambda_{2}$ are, to a good approximation,

$$
\begin{aligned}
& \lambda_{1}=\frac{v_{\alpha} \dot{R}}{\varpi \rho_{i}(1-x) U^{2} c R}, \\
& \lambda_{2}=\frac{c}{\varpi \sigma \dot{R} v_{\alpha}} ;
\end{aligned}
$$

and here $\lambda_{1} \gg \lambda_{2}$. All this is true provided

$$
\frac{\dot{R}^{2}}{k T_{i} / m_{\mathrm{H}}} \gg \frac{4 \rho_{i} R(1-x)}{\sigma}
$$

By an earlier argument the right-hand side of condition (25) is of order $10^{-3}$; the condition is therefore obeyed if $\dot{R}$ is not less than $1 / 30$ of the thermal velocity in the Hil region.

It appears that the radiation field is much more sharply limited on the side of negative $u$, the blue side. Very few photons reach large enough shifts on that side to escape there; virtually all will eventually escape with red shifts. On the red side the frequency distribution of the trapped photons is given by

$$
J(u) \propto \exp \left\{-\frac{c u^{3}}{3 \varpi \sigma \dot{R} v_{\alpha}}\right\} .
$$

The line thus becomes quite flat near $u=0$; the same also is true on the side of negative $u$. The mean value of $u$ at which a photon escapes (neglecting the few that escape on the blue side) is

$$
\bar{u}=\frac{\int_{0}^{\infty} u F(u) \mathrm{d} u}{\int_{0}^{\infty} F(u) \mathrm{d} u}
$$




$$
\begin{aligned}
& \int_{0}^{\infty} u^{3} \exp \left\{-c u^{3} / 3 \varpi \sigma \dot{R} v_{\alpha}\right\} \mathrm{d} u \\
= & \int_{0}^{\infty} u^{2} \exp \left\{-c u^{3} / 3 \varpi \sigma \dot{R} v_{\alpha}\right\} \mathrm{d} u \\
= & 1 \cdot 8\left(\frac{\varpi \sigma \dot{R} v_{\alpha}}{c}\right)^{1 / 3}=5 \cdot 7 \times 10^{11}(\sigma \dot{R})^{1 / 3} ;
\end{aligned}
$$

with $\sigma=10^{-2} \mathrm{gm} / \mathrm{cm}^{2}$ and $\dot{R}=10^{6} \mathrm{~cm} / \mathrm{sec}$ as typical values

$$
\bar{u}=1 \cdot 2 \times 10^{13} \mathrm{~s}^{-1} .
$$

Since $\vec{u} / v_{\alpha}$ is of order $0.5 \%$ the nebula expands about $0.5 \%$ during the period that a typical photon remains trapped. Note that $\bar{u}$ is more than 2 orders of magnitude larger than the thermal width $U$. This confirms that the Ly- $\alpha$ line spreads far out into the wings before its photons can escape from the nebula.

\section{The Mechanical Effect of Radiation Pressure}

It has now been shown that $\mathrm{Ly}-\alpha$ radiation is generated in the ionized region of the nebula at a rate $0.3 L_{*} \mathrm{erg} / \mathrm{sec}$, and that it loses a fraction $\bar{u} / v_{\alpha}$ of its original energy to the expanding shell of non-ionized gas. Thus the rate at which work is done on the shell is

$$
M \dot{R} \ddot{R}=0 \cdot 3 L_{*} \bar{u} / v_{\alpha} \equiv 0 \cdot 3 L_{*} \times 1 \cdot 8\left(\frac{\varpi M \dot{R}}{4 \pi R^{2} c v_{\alpha}^{2}}\right)^{1 / 3}
$$

or

$$
R^{2 / 3} \dot{R}^{2 / 3} \ddot{R}=2 \times 10^{-5} L_{*} / M^{2 / 3}
$$

To make a numerical estimate assume first that $L_{*}$ is constant and $R$ has the form $a t^{\mathrm{s}}$. Substitution shows that

$$
R=2 \cdot 2 \times 10^{-2} \frac{L_{*}^{3 / 7}}{M^{2 / 7}} t^{8 / 7}
$$

and

$$
\dot{R}=2 \cdot 5 \times 10^{-2} \frac{L_{*}^{3 / 7}}{M^{2 / 7}} t^{1 / 7}
$$

In this case most of the acceleration occurs when $t$ is small. Later the nebula expands at almost a constant rate. For example, if $L_{*}=10^{37} \mathrm{erg} / \mathrm{sec}$ and $M=10^{33} \mathrm{gm}$, then

$$
\dot{R}=2.5 \times 10^{6} \mathrm{~cm} / \mathrm{sec} \text { and } R=2 \cdot 2 \times 10^{17} \mathrm{~cm} \text {, }
$$


when $t=10^{11} \mathrm{sec}$. For $t$ larger or smaller by a factor 10 , the estimate for $R$ changes by only $45 \%$ upwards or downwards.

Solutions in which $R$ varies like a power of $t$ can also be obtained when the luminosity $L_{*}$ is assumed to vary with time. This assumption is more reasonable, for observation seems to show that the typical central star evolves with increasing luminosity early in the life of a nebula (Seaton, 1966). To illustrate this case, let $L_{*}=l_{*} t^{2}$, and take $l_{*}=10^{15} \mathrm{erg} / \mathrm{s}^{3}$, so that $L_{*}=10^{37} \mathrm{erg} / \mathrm{sec}$ when $t=10^{11} \mathrm{sec}$. Equation (30) is now solved by

$$
R=\alpha t^{2} \quad \text { and } \quad \dot{R}=2 \alpha t,
$$

where $\alpha=6 \times 10^{-3} l_{*}^{3 / 7} / M^{2 / 7} \approx 6 \times 10^{-6} \mathrm{~cm} / \mathrm{s}^{2}$.

At time $t=10^{11} \mathrm{sec}$ the predicted values for $R$ and $\dot{R}$ are somewhat smaller than for the case in which $L_{*}$ is constant. This is as expected. For the time $t=2 \times 10^{11} \mathrm{sec}$, Equations (34) predict $R=2.4 \times 10^{17} \mathrm{~cm}$ and $\dot{R}=2.4 \times 10^{6} \mathrm{~cm} / \mathrm{sec}$, but by then some of the approximations made earlier are beginning to fail. The luminosity $L_{*}$ would equal $4 \times 10^{37} \mathrm{erg} / \mathrm{sec}$ at that stage, and, according to Equation (3), the mass $M_{i}$ in the HII region is about $6 \times 10^{32} \mathrm{gm}$. An appreciable fraction of the shell has therefore been ionized, and the equations used here will cease to be valid. In fact the condition that $M_{i}$ be small compared with $M$, the initial mass of the shell, is

$$
2 \cdot 6 \times 10^{5} \frac{l_{*} t^{2}}{\beta^{1 / 2}} m_{\mathrm{H}} R^{3 / 2} \ll M ;
$$

with $R$ expressed in terms of $t$, by Equation (34), the inequality becomes

or $t \ll 2 \cdot 2 \times 10^{11} \mathrm{sec}$.

$$
t^{4} \ll \frac{M^{10 / 7} \beta^{1 / 2}}{123 m_{\mathrm{H}} l_{*}^{8 / 7}} \approx 2 \times 10^{45},
$$

The shell of atomic hydrogen ceases to exist after about $2 \cdot 2 \times 10^{11} \mathrm{sec}$. If our formulae were valid until then they would predict for that time a radius of $3 \times 10^{17} \mathrm{~cm}$ and a velocity $2.6 \times 10^{6} \mathrm{~cm} / \mathrm{sec}$, about twice the speed of sound in the HII region. The shell is then moving so fast that one cannot be sure that the ionized gas will fill the HII region uniformly. Nevertheless a straightforward comparison according to the present calculation shows that the radiation pressure and gas pressure become equally strong rather earlier than this time. At time $t$ one finds that the radiation pressure is

and the gas pressure

$$
p_{R}=\frac{M \ddot{R}}{4 \pi R^{2}} \approx 27 \frac{M^{9 / 7}}{l_{*}^{3 / 7}} t^{-4},
$$

$$
p_{G}=\frac{2\left(M_{i} / m_{\mathrm{H}}\right)}{(4 \pi / 3) R^{3}} k T_{i} \approx 10^{3} \frac{M^{3 / 7}}{l_{*}^{1 / 7}} t^{-2}
$$


so that $p_{R}$ much exceeds $p_{G}$ as long as

$$
t \ll 0 \cdot 16\left(\frac{M^{3}}{l_{*}}\right)^{1 / 7} \approx 1.6 \times 10^{11} .
$$

At $t=1 \cdot 6 \times 10^{11} \mathrm{sec}$ the estimated velocity of the shell is

$$
\dot{R}=2 \times 10^{-3} M^{1 / 7} l_{*}^{2 / 7} \approx 2 \times 10^{6} \mathrm{~cm} / \mathrm{sec} .
$$

Thus the radiation pressure $p_{R}$ provides the initial acceleration, and makes the shell expand faster than the sonic speed in the ionized gas, before the gas pressure $p_{G}$ can become as important as $p_{R}$. Soon after $p_{G}$ begins to be significant the shell will disappear, in any case.

\section{Pressure Exerted at the Ionization Front}

There will also be dynamical effects at the ionization front which may influence the motion of the outer shell. Conditions at ionization fronts (or I-fronts) have often been discussed, in the context of the interstellar medium (Axford, 1961; Mathews, 1965; Hjellming, 1966). Only some order-of-magnitude estimates will be made in this section.

Briefly mass, momentum and energy must be conserved at the I-front, and these requirements are expressed by three conservation laws for

$$
\begin{array}{ll}
\text { mass flux } & \Phi=\rho_{0} v_{0}=\rho v \\
\text { momentum flux } & \Pi=p_{0}+\rho_{0} v_{0}^{2}=p+\rho v^{2} \\
\text { energy conservation } & 2 \mathscr{E}+\frac{5 p_{0}}{\rho_{0}}+v_{0}^{2}=\frac{5 p}{\rho}+v^{2} .
\end{array}
$$

Here $p$ is the pressure, $\rho$ the density and $v$ the speed relative to the I-front. $\mathscr{E}$ is the net thermal energy gain, per unit mass, due to the ionization process (possibly allowing for subsequent cooling). The suffixes zero refer to the non-ionized gas ahead. If the front advances slowly relative to that gas and the temperature there is low, then $\left(5 p_{0} / \rho_{0}\right)+v_{0}^{2}$ is negligible compared with $2 \mathscr{E}$. To estimate a lower bound to the pressure ahead, one looks for the minimum value of $\Pi$, given $\Phi$ and $\mathscr{E}$. Provided $v_{0}^{2}$ is small compared with $p_{0} / \rho_{0}$, that is provided the I-front moves at low speed into the gas ahead, $p_{0}$ will approximately equal $\Pi$. With a little algebra one finds that

$$
p_{0} \approx \Pi=\frac{\Phi}{v}\left(\frac{2}{5} \mathscr{E}+\frac{4}{5} v^{2}\right) \geqslant \frac{4 \sqrt{2}}{5} \Phi \mathscr{E}^{1 / 2} .
$$

The rate of flow of mass into the Hil region, per unit surface area, is

$$
\Phi=\frac{\dot{M}_{i}}{4 \pi R^{2}} \approx \frac{10^{6} l_{*}^{2 / 7} M^{1 / 7} m_{\mathrm{H}}}{\beta^{1 / 2} t},
$$


after substituting from Equations (3) and (34), and putting $L_{*}=l_{*} t$. Further $\mathscr{E} \approx k T_{*} / m_{\mathrm{H}}$, where $T_{*}$ is the surface temperature of the central star, and it follows from Equations (42) and (43) that

$$
p_{0} \gtrsim \frac{10^{6} l_{*}^{2 / 7} M^{1 / 7}}{\beta^{1 / 2}}\left(k T_{*} m_{\mathrm{H}}\right)^{1 / 2} t^{-1}
$$

Putting $T_{*}=10^{5}{ }^{\circ} \mathrm{K}$, and on substitution for $\beta$, the pressure $p_{I}$ at the ionization front will be found to satisfy

so that $p_{I} \gg p_{R}$ when

$$
p_{I}\left(\equiv p_{0}\right) \gtrsim 10^{-5} l_{*}^{2 / 7} M^{1 / 7} / t
$$

$$
\begin{aligned}
10^{-5} l_{*}^{2 / 7} M^{1 / 7} & \gg 27 \frac{M^{9 / 7}}{l_{*}^{3 / 7}} t^{-4}, \quad \text { or } \\
t^{3} & \gg 27 \times 10^{6} \frac{M^{8 / 7}}{l_{*}^{5 / 7}}=2.5 \times 10^{33}, \text { or } \\
t & \gg 1.4 \times 10^{11}
\end{aligned}
$$

Thus $p_{G}$ and $p_{I}$ begin to dominate $p_{R}$ at about the same time. But note that $p_{G}$ is then decreasing more rapidly than $p_{I}$.

\section{Discussion}

In the paper the description of the dynamics of a planetary nebula has been drastically simplified. Even so it seems clear that radiation pressure in Lyman- $\alpha$ will be very important, until the speed of expansion becomes too high. During this phase the nebula is expected to contain a large proportion of non-ionized hydrogen. After about $1.5 \times 10^{11} \mathrm{sec}$ the recoil pressure at the ionization front begins to be more important than radiation pressure and will dominate the motion, until the hydrogen has been ionized.

From a study of photographs it is quite obvious that spherical symmetry is not a common property among planetary nebulae (see, e.g., Henize, 1967; Westerlund and Henize, 1967; Khromov and Kohoutek, 1968; Perek and Kohoutek, 1967). Indeed the typical nebula is more likely to be ring-like, or, when seen sideways on, butterflyshaped. There seem to be two ways in which this different symmetry might be introduced by the dynamical processes described in this paper.

One possibility is that the original, non-ionized nebula was an oblate spheroid. The atomic hydrogen shell formed from such a nebula would contain less mass per unit area over the poles, that is at the ends of the short axis of the spheroid. The polar parts of the shell would let through a greater flux of radiation and they would therefore be accelerated to a higher speed. Eventually there would be left behind a ring-like region, which expands more slowly, and has a higher density. 
Alternatively, the primitive nebula might have been spherically symmetrical, with a central star that rotates fast. The stellar surface temperature is then a function of latitude, with hot regions near the poles, and cooler regions over the equator. In the early evolution of the nebula the uneven distribution of colour and luminosity over the star would probably have little influence on the manner in which radiation pressure repels the shell, since Ly- $\alpha$ photons undergo many scatterings, and presumably redistribute themselves in a symmetrical fashion. But later the ionization front would advance faster into the polar parts of the non-ionized shell, and the recoil pressure there would be greater. These parts of the shell would therefore be expelled with a higher speed, once again leaving a ring-like structure behind. It is possible that these rings would appear to be butterfly-shaped, when seen sideways on.

Finally a closer inspection of the photographs show that planetary nebulae also have a rather finely detailed filamentary structure. No doubt there is some dynamical mechanism, like a Rayleigh-Taylor instability, which gives rise to these features. Such problems need to be studied much more thoroughly.

\section{References}

Aller, L.H. (1956) Gaseous Nebulae, Chapman and Hall, London, p. 74.

Axford, W.I. (1961) Phil. Trans. R. Soc. Lond., A253, 301.

Gebbie, K. B., Seaton, M.J. (1963) Nature, 199, 580.

Harman, R.J., Seaton, M.J. (1966) Mon. Not. R. astr. Soc., 132, 15.

Henize, K.G. (1967) Astrophys. J. Suppl. Ser., No. 126.

Hjellming, R. M. (1966) Astrophys. J., 143, 420.

Khromov, G.S., Kohoutek, L. (1968) in the present volume, p. 227.

Mathews, W.G. (1965) Astrophys. J., 142, 1120.

Perek, L., Kohoutek, L. (1967) Catalogue of Galactic Planetary Nebulae, Academia, Prague.

Seaton, M.J. (1966) Mon. Not. R. astr. Soc., 132, 113.

Westerlund, B. E., Henize, K.G. (1967) Astrophys. J. Suppl. Ser., 126.

\section{DISCUSSION}

Underhill: How do you get the original neutral gas nebula around the star in order to start the process?

Menon: Is it implied that the neutral hydrogen shell was originally part of the star and not a part of the interstellar medium?

Kahn: One picture of the early evolution of a planetary nebula is to imagine that in the beginning a cloud of non-ionized gas surrounds the central star. The star then becomes hot and begins to create

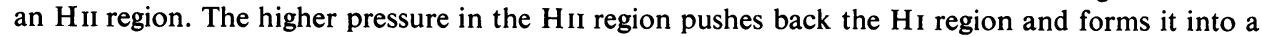
thin shell. According to this view it is almost necessary that an $\mathrm{H}_{\mathrm{I}}$ shell should surround the nebula at some stage.

Evans: This is a very attractive idea because of the frequency with which one observes a breakdown of spherical symmetry in the sense of a transition to a bipolar form. One example is Henize 150 where the ring has broken at opposite points and gas streams out, being re-excited by the central star as it crosses the beam.

Böhm: In the second phase of the central stars' evolution their luminosity decreases with time. How does this affect the development of the ionization front?

Kahn: While the non-ionized shell is still in existence, any reduction in the Lyman continuum flux 
at the I-front slows down the flow through the front. If the radiation from the star gets so weak that no ionizing radiation gets through the $\mathrm{H}$ II region to the $\mathrm{I}-\mathrm{front}$, then a recombination front will form.

Gurzadian: All these considerations are very interesting, but your theory should take into account the role of magnetic fields which might be present in the nebula.

Kahn: It is most doubtful whether one can reasonably assume that a magnetic field can exist which is strong enough to affect the fluid motions significantly.

Capriotti: The work of Kahn is quite pertinent to the study of the planetary nebulae. One way to test the validity of the assumption that the $\mathrm{He}{ }^{3} \mathrm{~S}$ state is depopulated through photo-ionization by $L y-\alpha$ radiation is to estimate the length of time for which a sufficiently high Ly- $\alpha$ density can be sustained. The blanketing effect of a thin shell of neutral hydrogen has been proposed as the agent for the trapping of $L y-\alpha$ radiation to the extent that it depopulates the $2^{3} S$ state at the observed rate. Kahn's result that this blanketing effect could last for only a very short time is a reason for rejecting this proposed mechanism.

Underhill: Kahn's remarks referred to the initial stages of development of a planetary nebula. Reeves has already suggested that the injection of low-energy protons might be a significant factor in causing the observed line spectrum of the nebula. Would not the original state of the nebula then be quite different from the state it would have if only radiation processes were occurring? In such a case the relaxation to the state which we observe might differ from that postulated by the types of theory discussed today (i.e. chiefly ionization by stellar ultraviolet and Ly- $\alpha$ and $\mathrm{He}$ II 303 lines). A continuous ejection of protons at $2000 \mathrm{~km} / \mathrm{sec}$ from the central star is not unexpected if we extrapolate from the known behavior of Wolf-Rayet stars and O-type supergiants.

Münch: I wish to express the point of view held by Capriotti in somewhat different words. The need for a deactivation agent of the $\mathrm{He}$ I $2^{3} \mathrm{~S}$ level arose from the considerations regarding the nebular absorption of $\mathrm{HeI}_{\mathrm{I}} \lambda 3889$ in stars imbedded in HII regions made by Wilson and myself. We found that considering two-photon decay, collisional deactivation, and photo-ionization by star light only, the observed absorption implied a very small emission measure, in disagreement with observation. On this basis I suggested that photo-ionization by Ly- $\alpha$ may play a role. The upward revision of the collisional deactivation rates reported at this conference by Seaton will help the situation, but will by no means completely explain the observed population of HeI ${ }^{3} \mathrm{~S}$. The lack of $\lambda 3889$ absorption in many stars imbedded in HII regions (and I have observed more than 100 of them) appears to me to be a serious problem.

Capriotti: The electron density in an HII region is probably low enough so that the $\lambda 10830 / \lambda 5876$ line-intensity ratio is near the low-density limit. Therefore, one probably would not be able to use this particular ratio in order to estimate the relative population density of the $2^{3} \mathrm{~S}$ state and check on the results obtained by emission measures in $\lambda 3889$. On the other hand, because of the relatively low electron densities in the $\mathrm{H}$ II regions the deviation of the depopulation rate of the $2^{3} \mathrm{~S}$ state as determined by a $\lambda 3889$ emission measure from a theoretically predicted rate could hardly be blamed on underestimates of the collision cross-sections for $2^{3} \mathrm{~S} \rightarrow 2^{1} \mathrm{~S}, 2^{1} \mathrm{P}$, and ${ }^{1} \mathrm{~S}$ transitions. It would be nice if the discrepancy between theory and observation as regards the depopulation of the $2^{3} \mathrm{~S}$ state of helium in both HII regions and the planetary nebulae could be explained on the same basis. 